\title{
DUST COMPOSITION, ENERGETICS, AND MORPHOLOGY OF THE GALACTIC CENTER
}

\author{
KIN-WING CHAN \\ NASA/Ames Research Center \\ MS 245-6, Moffett Field, CA 94035, USA
}

S. H. MOSELEY

NASA/Goddard Space Flight Center

Code 685, Greenbelt, MD 20771, USA

S. CASEY

Hughes/STX/NASA/Goddard Space Flight Center

Code 685, Greenbelt, MD 20771, USA

J. P. HARRINGTON

University of Maryland

Dept. of Astronomy, College Park, MD 20742, USA

E. DWEK

NASA/Goddard Space Flight Center

Code 685, Greenbelt, MD 20771, USA

R. LOEWENSTEIN

Yerkes Observatory

William Bay, Wisconsin, WI 53191, USA

F. VÁROSI

Hughes/STX/NASA/Goddard Space Flight Center

Code 685, Greenbelt, MD 20771, USA

\section{AND}

W. GLACCUM

Applied Research Corporation

8201 Corporate Drive, Landover, MD 20785, USA

\footnotetext{
Abstract. Spectra at $16-45 \mu \mathrm{m}$ of several regions within the central $80^{\prime \prime}$ of the Galaxy have been obtained at $20^{\prime \prime}$ resolution using the Goddard Cryogenic Grating Spectrometer No. 2 on the Kuiper Airborne Observatory. A 
broad band of excess emission extending from 24 to $45 \mu \mathrm{m}$ is present in the spectra at positions covering the "tongue" and the inner edge of the circumnuclear disk. A similar dust emission feature has been observed in some carbon-rich evolved stars and in a nitrogen-rich evolved massive star. The observations reported here are the first detection of this dust emission feature in the interstellar medium. After considering several possible candidates of the carrier for this $30 \mu \mathrm{m}$ dust feature, we find that $\mathrm{MgS}$ is the best owing to its good fit to the observed spectra. The origin of this $30 \mu \mathrm{m}$ feature in the Galactic center is unknown. Based on the theoretical results of dust condensation and elemental abundances in a supernova, we find that the supernovae in the central $500 \mathrm{pc}$ could provide the amount of $\mathrm{MgS}$ dust, which we proposed as the carrier of the $30 \mu \mathrm{m}$ dust feature, observed in the central $3 \mathrm{pc}$.

We find that the dust properties in the "tongue" and the inner edge of the circumnuclear disk are different from the dust in the cavity, which shows no evidence for the $\sim 30 \mu \mathrm{m}$ feature in its spectrum. The relation between the tongue feature and the inner disk has been a subject of considerable debate, with suggestions that the tongue material is either falling in or has been expelled from the Galactic center. Our observations suggest that if there is a physical connection between the two regions, then the tongue material is more likely falling in toward the Galactic center.

Two kinds of dust composition and corresponding heating sources are argued to be responsible for the dust emission in the Galactic center: (1) the hot dust component $(140-300 \mathrm{~K})$ heated by IRS sources that are distributed throughout the cavity; and (2) the warm dust component (60 - $90 \mathrm{~K}$ ) heated by a He I emission-line star cluster that give rise to a local interstellar radiation field that heats the dust in the central several parsecs. We find a lower limit of $\sim 1 \times 10^{6} \mathrm{~L}_{\odot}$ for the He I emission-line star cluster.

Finally, we find that the line-of-sight extinction across the cavity is not uniform. It is diminished and roughly constant in the northeastern side of the cavity but increases to the southwestern side. Based on this result, together with the 1995 study by Zylka et al. who found that there is a cold dust component along the line of sight to the central $30^{\prime \prime}$ of the Galaxy, we propose that there is a cool dust envelope with nonuniform distribution covering the cavity of the Galactic center. 